\title{
Theory of deep impurity levels in $\mathrm{CuCl}$
}

\author{
Shang-Yuan Ren,* Roland E. Allen, ${ }^{\dagger}$ and John D. Dow \\ Department of Physics and Materials Research Laboratory, \\ University of Illinois at Urbana-Champaign, Urbana, Illinois 61801
}

\author{
I. Lefkowitz \\ U. S. Army Research Office, Research Triangle Park, North Carolina 22709 \\ and Hunter College of the City University of New York, New York 10021 \\ and University of North Carolina, Chapel Hill, North Carolina 27514 \\ (Received 11 June 1981)
}

\begin{abstract}
The theory of deep impurity levels is extended to semiconductors with $d$ electrons. The major chemical trends are predicted for a large number of substitutional impurities in $\mathrm{CuCl}$. Deep levels are found for $\mathrm{S}$ and $\mathrm{Se}$ impurities on the $\mathrm{Cl}$ site, but not for $\mathrm{Ag}$ or $\mathrm{Au}$ on the $\mathrm{Cu}$ site, in agreement with experiment. The theory also predicts no deep level for isolated $\mathrm{O}$ on the $\mathrm{Cl}$ site, thus supporting the conclusion that the observed O-related defect is not a simple substitutional impurity.
\end{abstract}

\section{INTRODUCTION}

$\mathrm{CuCl}$ has been reported to exhibit an anomalous diamagnetism that is reminiscent of superconductivity $^{1-3}$ and possibly associated with defects. ${ }^{4}$ The importance of defect states is further emphasized by the occurrence of a broadened $z_{3}$ exciton line $e^{5}$ in the same temperature region in which the diamagnetism is observed. This broadening has been variously related to defect states, free electron effects, ${ }^{5}$ a defect-stabilized plasma state, ${ }^{6}$ or a modified phonon spectrum. ${ }^{4}$ In order to provide some theoretical guidance for experiments involving defects in $\mathrm{CuCl}^{4,7}$ we have extended the theory of substitutional impurities ${ }^{8-10}$ to include $d$ electrons on the cation site. We focus on the deep levels-i.e., the levels that are bound within the fundamental band gap predominantly by the central-cell defect potential.

The theory of impurities in $s p^{3}$-bonded covalent materials, as enunciated by Hjalmarson et al. ${ }^{8}$ contains two basic ingredients: (i) an empirical tightbinding model of the host electronic structure that incorporates experimental information, chemical trends, and the relevant features of theoretical energy bands, and (ii) a defect potential whose offdiagonal matrix elements in the tight-binding basis are determined by Harrison's $d^{-2}$ scaling rule ${ }^{11}$ and whose diagonal matrix elements $V_{l}$ are determined by the differences in impurity and host atomic energies:

$$
V_{l}=\beta_{l}\left(E_{l}^{i}-E_{l}^{h}\right)
$$

Here $E_{l}^{i}$ and $E_{l}^{h}$ are impurity and host orbital energies for states of angular momentum $l$, and are converted into "orbital energies within the solid" by the constant factor $\beta_{l}$.

In this paper, we generalize the theory of $\mathrm{Hjal}$ marson et al. to include $d$-electron orbitals on the cation site. This immediately leads to two changes: (i) The host Hamiltonian $H_{0}$ involves five $d$ orbitals on the cation site, as well as one $s$ orbital and three $p$ orbitals on each site, and (ii) the defect potential matrix for $\mathrm{Cu}$ substitutional impurities is also augmented by matrix elements involving $d$ orbitals.

\section{THE MODEL}

Our 13-state tight-binding host Hamiltonian is shown in Appendix A. Its parameters, given in Table I, were determined by a fitting procedure described in Appendix B. The resulting band structure is displayed in Fig. 1, together with the results of a psuedopotential calculation by Kleinman and Mednick. ${ }^{12}$ The bands of Kunz et al. ${ }^{13}$ and of Zunger and Cohen ${ }^{14}$ are similar to those of Kleinman and Mednick.

The primary requirement of a successful tightbinding model is that it adequately reproduces the projected densities of states-not just the energy bands. It is these densities of states, for anion $s$ and $p$ electrons and for cation $s, p$, and $d$ electrons, that determine the defect levels in accordance with Eqs. (3.2) and (3.3). We imposed the following re- 
TABLE I. Parameters of the tight-binding model in eV. With these parameters the charges on the $\mathrm{Cu}$ and $\mathrm{Cl}$ ions are, respectively, $+0.59|e|$ and $-0.59|e|$.

\begin{tabular}{|c|c|c|c|c|c|c|c|}
\hline$E(s, c)$ & $E(s, a)$ & $E(p, c)$ & $E(p, a)$ & $E(d, c)$ & $E^{\prime}(d, c)$ & $V\left(x_{c}, y z_{c}\right)$ & \\
\hline 2.80 & -15.15 & 9.00 & -3.75 & -1.25 & -1.90 & 0 & \\
\hline$V(s, s)$ & $V\left(x_{a}, s_{c}\right)$ & $V\left(s_{a}, x_{c}\right)$ & $V(x, x)$ & $V(x, y)$ & $V_{s d \sigma}$ & $V_{p d \sigma}$ & $V_{p d \pi}$ \\
\hline-2.877 & 4.841 & 2.866 & 0 & 0 & -1.980 & -5.085 & 1.220 \\
\hline
\end{tabular}

quirements in fitting our tight-binding model: (i) The band gap is $3.25 \mathrm{eV} .{ }^{15}$ (ii) The $\mathrm{Cu} 3 d$ bands lie above the $\mathrm{Cl} 3 p$ bands, ${ }^{16}$ and the widths of these bands ${ }^{17}$ are approximately the observed widths.

(iii) The four main peaks observed in $\mathrm{x}$-ray photoemission ${ }^{16}$ have their observed positions. (iv) The top of the highest valence band is $75 \% \mathrm{Cu} 3 d$ and $25 \% \mathrm{Cl} 3 p .^{16}$ (v) The charge on the $\mathrm{Cu}$ ion is positive. (vi) The remaining important features in the pseudopotential bands of Refs. $12-14$ are adequately reproduced.

The resulting tight-binding Hamiltonian yields densities of state for the upper valence bands that are in good agreement with the available photoemission data, as shown in Table II and Fig. 2. It also produces bands that are in reasonable agreement with the pseudopotential calculations (Fig. 1).

\section{METHOD OF CALCULATION}

The change in the tight-binding Hamiltonian resulting from the introduction of an impurity or vacancy is the defect potential $V \equiv H-H_{0}$. If we neglect lattice relaxation, the defect potential is diagonal ${ }^{8}$ because Harrison's scaling rules ${ }^{11}$ imply that the off-diagonal matrix elements of $H$ and $H_{0}$ are equal.

Following the approach of Hjalmarson et al., we take the on-site diagonal defect potential for isoelectronic defects to be given by Eq. (1.1). ${ }^{18}$ The Hjalmarson et al. model was originally developed for studying isoelectronic defects in highly covalent materials; its application to charged centers in more ionic materials requires a modification of the expression for $V$ to account for the different ionic

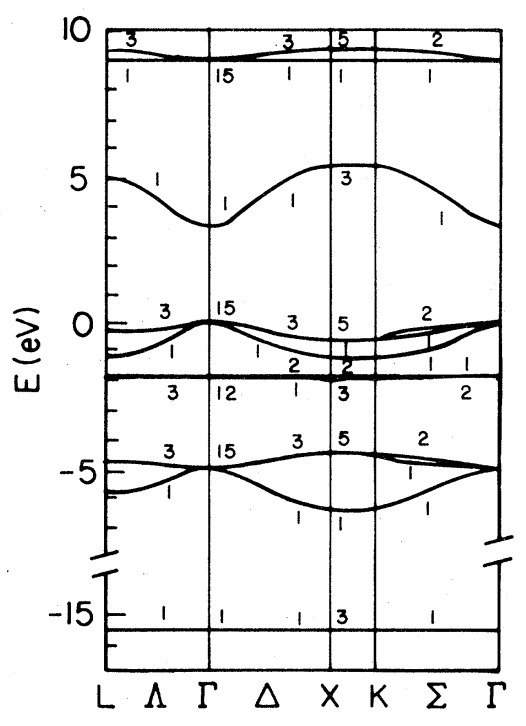

(a)

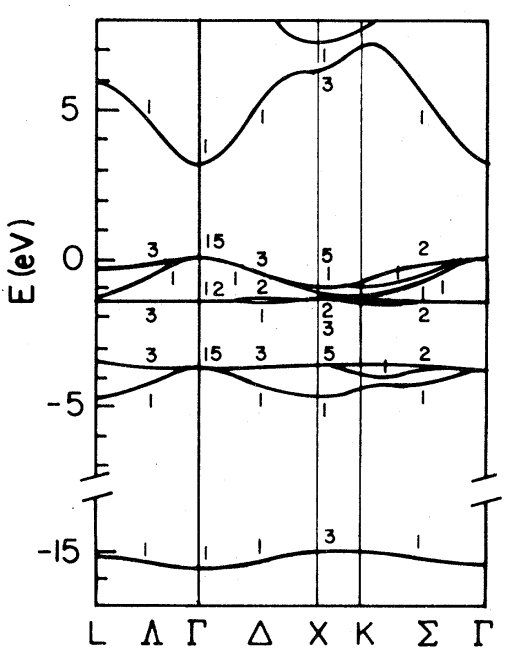

(b)

FIG. 1. Band structure of $\mathrm{CuCl}$ : (a) calculated from the Hamiltonian of Appendix A and Table I, (b) calculated by Kleinman and Mednick (Ref. 12). 
TABLE II. Experimental features fit in determining the empirical $\mathrm{CuCl} \mathrm{Hamiltonian.}$

\begin{tabular}{|c|c|c|}
\hline & Experiment & Theory \\
\hline Order of valence bands & $\begin{array}{l}\mathrm{Cu} 3 d \text { higher } \\
\text { than } \mathrm{Cl} 3 p^{\mathrm{a}}\end{array}$ & $\begin{array}{c}\mathrm{Cu} 3 d \text { higher } \\
\text { than } \mathrm{Cl} 3 p\end{array}$ \\
\hline $\begin{array}{c}\mathrm{Cu} 3 d \text { character at top of } \\
\text { valence band }\end{array}$ & $75 \%$ b & $75 \%$ \\
\hline $\begin{array}{c}\mathrm{Cl} 3 p \text { character at top of } \\
\text { valence band }\end{array}$ & $25 \%{ }^{b}$ & $25 \%$ \\
\hline Band gap $(\mathrm{eV})$ & $3.25^{\mathrm{c}}$ & 3.25 \\
\hline \multicolumn{3}{|l|}{ Photoemission peaks $(\mathrm{eV})$} \\
\hline$A$ & $0.8-1.4^{\mathrm{b}}$ & 0.6 \\
\hline$B$ & $1.9-2.6^{\mathrm{b}}$ & 1.9 \\
\hline$C$ & $4.9-5.2^{b}$ & 4.8 \\
\hline$D$ & $6.0-6.3^{b}$ & 6.4 \\
\hline Width of lower band $(\mathrm{eV})(\mathrm{Cl} 3 p)$ & $2.4^{\mathrm{a}}$ & 2.4 \\
\hline Width of upper band $(\mathrm{eV})(\mathrm{Cu} 3 d)$ & $1.7^{\mathrm{a}}$ & 2.0 \\
\hline
\end{tabular}

${ }^{\text {a }}$ Reference 17.

${ }^{\mathrm{b}}$ Reference 16.

${ }^{\mathrm{c}}$ Reference 15.

charges. This modification we term $\Delta V_{l}$; it is expected to have the same sign as the correction Eq. (1.1) would experience if ionic energies were used in place of atomic energies. Crude estimates of $\Delta V_{l}$ for singly charged defects in an ionic host such as $\mathrm{CuCl}$ indicate that it is of the order of 4 $\mathrm{eV} .{ }^{19}$ We shall see independently that a value of $\Delta V_{p}=3.5 \mathrm{eV}$ produces $\mathrm{S}$ and Se levels in agreement with the data.

The defect potential for an anion-site defect is thus a diagonal $4 \times 4$ matrix with one element $V_{s}$ and three elements $V_{p}$. For a cation-site defect, it is a $9 \times 9$ matrix with one element $V_{s}$, three ele-

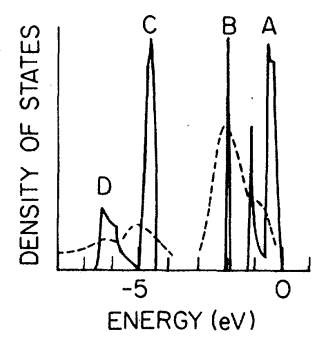

FIG. 2. Valence-band density of states calculated from the host Hamiltonian of this paper (solid curve), in comparison with the deconvoluted x-ray-photoelectronspectroscopy spectra obtained by $\mathrm{S}$. Kono et al. as cited in Ref. 16 (dashed curve). ments $V_{p}$, three elements $V_{d}$, and two elements $V_{d}^{\prime}$. (We do not assume that the $d_{x y}$ and $d_{y^{2}-z^{2}}$ defect potentials are equal.) In each case we have

$$
V_{l}=\beta_{l}\left(E_{l}^{i}-E_{l}^{h}\right)+\Delta V_{l} .
$$

Since the defect potential is localized, it is appropriate to use the Green's-function method to compute the defect energy levels. This method produces a secular equation in a localized basis that involves only those basis functions within the defect space $^{8-10}$ :

$$
\operatorname{det}\left[1-G_{0}(E) V\right]=0,
$$

where we have

$$
G_{0}(E) \equiv\left(E-H_{0}\right)^{-1} .
$$

For the anion site, this $4 \times 4$ problem reduces to four $1 \times 1$ problems, yielding one $A_{1}(s$-like) and three degenerate $T_{2}$ ( $p$-like) defect states. For the cation site, Eq. (3.2) is a $9 \times 9$ problem. Our decoupling of the cation $p$ and $d$ orbitals (see Appendix B) reduces Eq. (3.2) to nine scalar problems, yielding one $A_{1}$ ( $s$-like), three $T_{2}$ ( $p$-like), another three $T_{2}\left(d_{x y}\right.$-like $)$, and two $E\left(d_{y^{2}-z^{2}}\right.$-like $)$ states.

Each eigenvalue problem has the form

$$
\frac{1}{V_{\lambda b}}=G_{\lambda b}(E)=\int \frac{D_{\lambda b}\left(E^{\prime}\right) d E^{\prime}}{E-E^{\prime}}
$$


where

$$
\lambda=A_{1}(s), T_{2}(p), T_{2}\left(d_{x y}\right), \text { or } E\left(d_{y^{2}-z^{2}}\right),
$$

and $b$ means "anion" or "cation."

Here $D_{\lambda b}$ is the contribution of the $\lambda$-symmetric irreducible representation to the local density of states on the $b$ site:

$$
\begin{aligned}
D_{\lambda b}(E) & =\left\langle\lambda b\left|\delta\left(E-H_{0}\right)\right| \lambda b\right\rangle \\
& =\sum_{n \overrightarrow{\mathrm{k}}}\langle\lambda b \mid n \overrightarrow{\mathrm{k}}\rangle\langle n \overrightarrow{\mathrm{k}} \mid \lambda b\rangle \delta\left(E-E_{n \overrightarrow{\mathrm{k}}}\right) .
\end{aligned}
$$

\section{RESULTS}

The predictions for deep levels associated with isolated substitutional impurities are given in Figs. $3-8$. The predicted level for a given impurity is obtained from the intersection of the calculated curve with a vertical line representing the isoelectronic impurity potential.

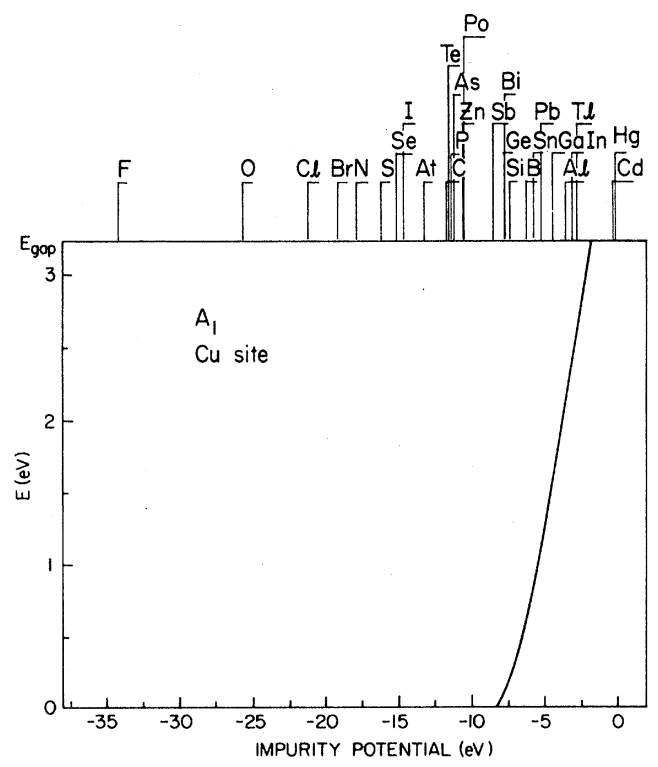

FIG. 3. Energies in $\mathrm{eV}$ of the $A_{1}$ ( $s$-like) substitutional defect levels predicted for impurities at the $\mathrm{Cu}$ site in $\mathrm{CuCl}$. The abscissa is the defect potential; each impurity has a tic at its potential. All transition-metal potentials are between -2 and $+2 \mathrm{eV}$; hence these defects are not predicted to produce $A_{1}$ deep traps. No $A_{1}$ deep levels are predicted to lie within the band gap for the isoelectronic impurities $\mathrm{Ag}$ ' and $\mathrm{Au}$.

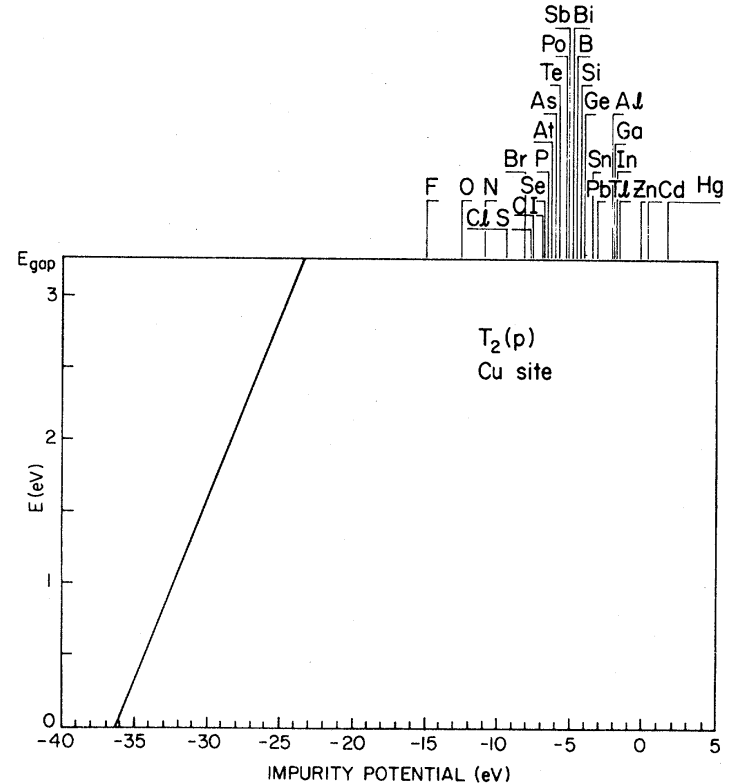

FIG. 4. Energies of the $T_{2}$ ( $p$-like) substitutional defect levels predicted for impurities at the $\mathrm{Cu}$ site in $\mathrm{CuCl} . T_{2} p$-derived levels in the gap are not predicted for any of the impurities.

These predictions should be viewed within the context of the goals of the theory. They represent a global view of the expected chemical trends in the defect data, and should be corrected for the different charge states of the defect levels, and, possibly, lattice relaxation. (The present calcula-

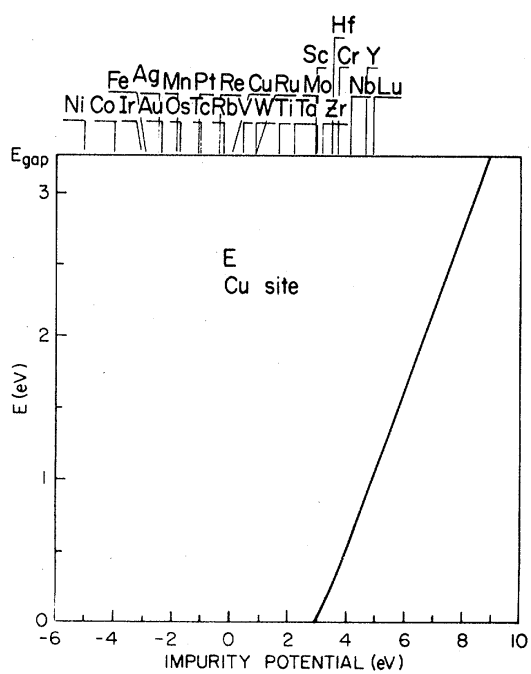

FIG. 5. Energies of the $E$-symmetric $\left(d_{y^{2}-z^{2}}-\right.$ like $)$ defect levels predicted for impurities at the $\mathrm{Cu}$ site in $\mathrm{CuCl}$. Notice that $\mathrm{Ag}$ and $\mathrm{Au}$ do not produce $d$-like $E$ defect levels in the gap when on the $\mathrm{Cu}$ site. 


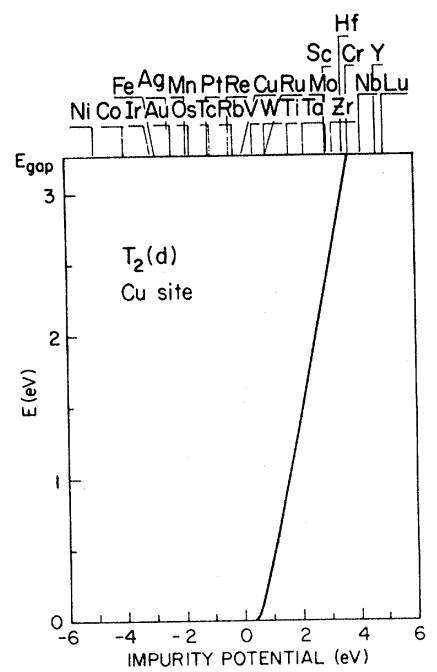

FIG. 6. $T_{2}$-symmetric $\left(d_{x y}\right.$-like $)$ defect levels for the $\mathrm{Cu}$ site. Note that $\mathrm{Ag}$ and $\mathrm{Au}$ again do not produce levels in the gap.

tions predict the neutral defect levels, and corrections to these levels for the different charge states can be estimated using simple electrostatic arguments.) Since the purpose of the theory is to predict trends, predictions of relative orderings of energy levels produced by different defects should be even more reliable than the theoretical energies of individual defects.

The predictions of the theory agree with the major experimental facts of which we are aware: (i) $\mathrm{S}$

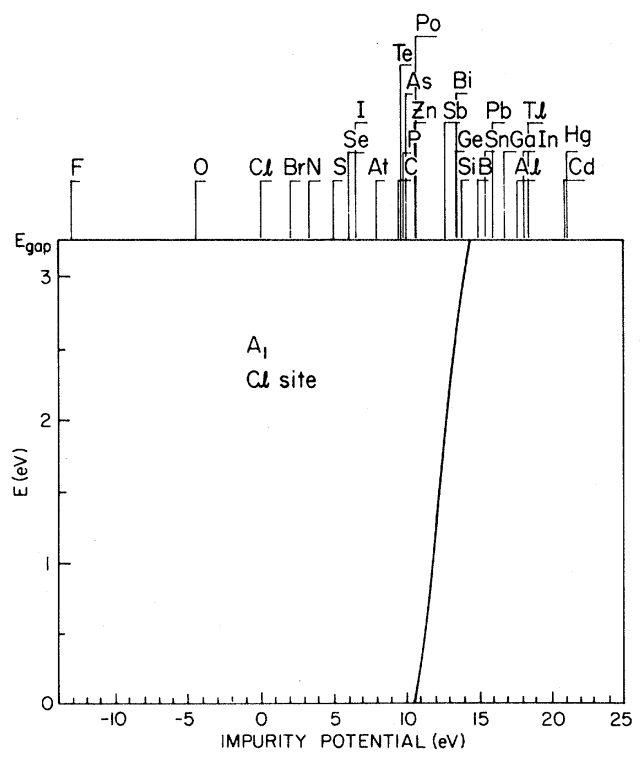

FIG. 7. Energies of the $A_{1}$ defect levels predicted for impurities on the $\mathrm{Cl}$ site of $\mathrm{CuCl}$.

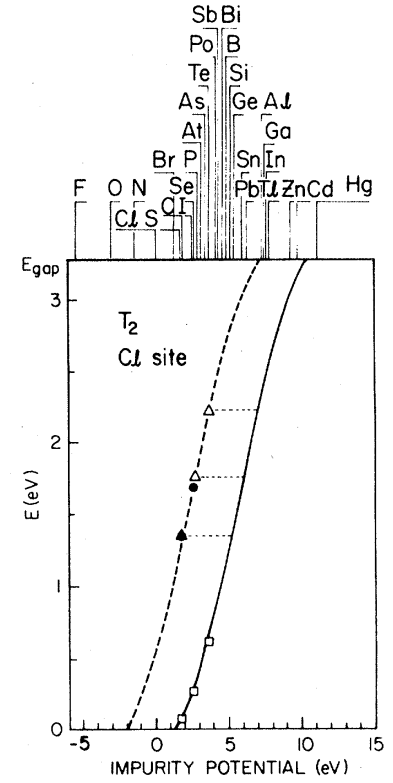

FIG. 8. Energies of the $T_{2}$ defect levels predicted for impurities on the $\mathrm{Cl}$ site of $\mathrm{CuCl}$. The solid line is the uncorrected theory; the points marked with open squares are the uncorrected predictions for $\mathrm{S}, \mathrm{Se}$ and Te levels. The solid circles represent the experimental results for $S$ and Se (after Ref. 20). The dashed line represents the corrected theory for acceptors, with the correction being determined by the $\mathrm{S}$ datum; the open triangles are the corrected predictions for $\mathrm{S}, \mathrm{Se}$, and Te.

and Se substituting for $\mathrm{Cl}$ are known to produce deep levels in $\mathrm{CuCl},{ }^{20,21}$ with the Se level being $0.32 \mathrm{eV}$ above the $\mathrm{S}$ level; (ii) searches for deep traps associated with $\mathrm{Ag}$ or $\mathrm{Au}$ substituting for $\mathrm{Cu}$ have been unsuccessfu1 ${ }^{22}$; and (iii) an O-related level has been identified ${ }^{20}$ which, unlike the $\mathrm{S}$ and Se levels, is not associated with the tetrahedral symmetry of a simple substitutional defect.

The experimental $\mathbf{S}$ and Se impurity levels have the same ordering as in the uncorrected predictions of Fig. 8, but are somewhat higher in absolute energy than predicted. As mentioned in Sec. III, the uncorrected predictions are for isoelectronic impurities [Eq. (1.1)], whereas the $\mathbf{S}$ and Se impurities are expected to have an extra electron in comparison with the replaced $\mathrm{Cl}$ atom. (Roughly speaking, $\mathrm{S}^{-}$and $\mathrm{Se}^{-}$replace $\mathrm{Cl}^{-}$.) Allowance for the fact that $\mathrm{S}$ is not isoelectronic to $\mathrm{Cl}$, through modification of the defect potential as prescribed in (3.1), brings the theory into improved agreement with the data. By choosing the defect potential modification $\Delta V_{p}$ such that the $\mathrm{S}$ datum is reproduced, we find a reasonable value $\Delta V_{p}=3.5 \mathrm{eV}$. The correction $\Delta V_{p}$ contains not only the atomistic 
correction discussed above, but the long-range polarization energy, which systematically alters the levels of the S, Se, and Te defects. We then predict a Se level in excellent agreement with the observed value, as indicated in Fig. 8. Such agreement is, of course, expected of a theory that is intended to predict trends in impurity levels more reliably than absolute energies. ${ }^{8}$

The theory predicts that the defect levels associated with $\mathrm{Ag}$ or $\mathrm{Au}$ on the $\mathrm{Cu}$ site all lie within the bands and outside of the fundamental band gap. Thus it explains why no deep levels have been observed for these impurities. ${ }^{22}$

An especially interesting prediction of the model is that isolated $\mathrm{O}$ on a $\mathrm{Cl}$ site should not produce a deep trap, but its isoelectronic mates $\mathbf{S}$ and $\mathrm{Se}$ should. The reason for this is that $\mathrm{O}$ attracts electrons more strongly than $\mathrm{Cl}$, whereas $\mathrm{S}$ and $\mathrm{Se}$ are weaker. The $\mathrm{O}$ levels should therefore lie below the corresponding $\mathrm{Cl}$ levels, outside the gap, whereas the $\mathrm{S}$ and Se levels should lie above $\mathrm{Cl}$, within the gap. Thus, in a natural way, the theory supports the interpretation that the O-related defect, with a level $1.08 \mathrm{eV}$ above the valence band, ${ }^{20}$ is not a substitutional impurity like $S$ and $\mathrm{Se}$, but is instead an extended defect of nontetrahedral symmetry. The present work, therefore, lends indirect support to the work of Kunz et al., ${ }^{13}$ who have proposed that a complex consisting of $\mathrm{O}^{-2}$ and a vacancy produces a level near the conduction-band edge.

Finally, when it substitutes for $\mathrm{Cl}$, the impurity Te should produce a $T_{2}$-symmetric defect level at about $2.20 \mathrm{eV}$ above the valence-band edge. Observation of this level would provide gratifying confirmation of the present theory.

\section{ACKNOWLEDGMENTS}

We thank O. F. Sankey for his many enlightening comments, and we acknowledge the Office of Naval Research (Grant No. ONR-N-00014-77-C0537) for support, and the Materials Research Laboratory for the use of their computer (Department of Energy Grant No. DE-AC02-76-ER01198).

\section{APPENDIX A}

The 13-orbital tight-binding Hamiltonian has the form as shown in Table III, where we have

$$
\begin{aligned}
& g_{0}=\cos q_{1} \cos q_{2} \cos q_{3}-i \sin q_{1} \sin q_{2} \sin q_{3} \\
& g_{1}=-\cos q_{1} \sin q_{2} \sin q_{3}+i \sin q_{1} \cos q_{2} \cos q_{3} \\
& g_{2}=-\sin q_{1} \cos q_{2} \sin q_{3}+i \cos q_{1} \sin q_{2} \cos q_{3} \\
& g_{3}=-\sin q_{1} \sin q_{2} \cos q_{3}+i \cos q_{1} \cos q_{2} \sin q_{3}
\end{aligned}
$$

with

$$
\overrightarrow{\mathrm{q}}=\frac{a}{4} \overrightarrow{\mathrm{k}}
$$

and

\begin{tabular}{|c|c|c|c|c|c|c|c|}
\hline & $s_{a}$ & $s_{c}$ & $x_{a}$ & $y_{a}$ & $z_{a}$ & $x_{c}$ & $y_{c}$ \\
\hline$s_{a}$ & $E(s, a)$ & $V(s, s) g_{0}^{*}$ & 0 & 0 & 0 & $-V\left(s_{a}, x_{c}\right) g_{1}^{*}$ & $-V\left(s_{a}, x_{c}\right) g_{2}^{*}$ \\
\hline$s_{c}$ & $V(s, s) g_{0}$ & $E(s, c)$ & $V\left(s_{c}, x_{a}\right) g_{1}$ & $V\left(s_{c}, x_{a}\right) g_{2}$ & $V\left(s_{c}, x_{a}\right) g_{3}$ & 0 & 0 \\
\hline$x_{a}$ & 0 & $V\left(s_{c}, x_{a}\right) g_{1}^{*}$ & $E(p, a)$ & 0 & 0 & $V(x, x) g_{0}^{*}$ & $V(x, y) g_{3}^{*}$ \\
\hline$y_{a}$ & 0 & $V\left(s_{c}, x_{a}\right) g_{2}^{*}$ & 0 & $E(p, a)$ & 0 & $V(x, y) g_{3}^{*}$ & $V(x, x) g_{0}^{*}$ \\
\hline$z_{a}$ & 0 & $V\left(s_{c}, x_{a}\right) g_{3}^{*}$ & 0 & 0 & $E(p, a)$ & $V(x, y) g_{2}^{*}$ & $V(x, y) g_{1}^{*}$ \\
\hline$x_{c}$ & $-V\left(s_{a}, x_{c}\right) g_{1}$ & 0 & $V(x, x) g_{0}$ & $V(x, y) g_{3}$ & $V(x, y) g_{2}$ & $E(p, c)$ & 0 \\
\hline$y_{c}$ & $-V\left(s_{a}, x_{c}\right) g_{2}$ & 0 & $V(x, y) g_{3}$ & $V(x, x) g_{0}$ & $V(x, y) g_{1}$ & 0 & $E(p, c)$ \\
\hline$z_{c}$ & $-V\left(s_{a}, x_{c}\right) g_{3}$ & 0 & $V(x, y) g_{2}$ & $V(x, y) g_{1}$ & $V(x, x) g_{0}$ & 0 & 0 \\
\hline$x_{c} y_{c}$ & $V\left(s_{a}, x y_{c}\right) g_{3}$ & 0 & $-V\left(x_{a}, x y_{c}\right) g_{2}$ & $-V\left(x_{a}, x y_{c}\right) g_{1}$ & $-V\left(x_{a}, y z_{c}\right) g_{0}$ & 0 & 0 \\
\hline$y_{c} z_{c}$ & $V\left(s_{a}, x y_{c}\right) g_{1}$ & 0 & $-V\left(x_{a}, y z_{c}\right) g_{0}$ & $-V\left(x_{a}, x y_{c}\right) g_{3}$ & $-V\left(x_{a}, x y_{c}\right) g_{2}$ & $V\left(x_{c}, y z_{c}\right)$ & 0 \\
\hline$x_{c} z_{c}$ & $V\left(s_{a}, x y_{c}\right) g_{2}$ & 0 & $-V\left(s_{a}, x y_{c}\right) g_{3}$ & $-V\left(x_{a}, y z_{c}\right) g_{0}$ & $-V\left(x_{a}, x y_{c}\right) g_{1}$ & 0 & $V\left(x_{c}, y z_{c}\right)$ \\
\hline$y_{c}^{2}-z_{c}^{2}$ & 0 & 0 & 0 & $-V\left(y_{a}, y^{2}-z_{c}^{2}\right) g_{2}$ & $V\left(y_{a}, y^{2}-z_{c}^{2}\right) g_{3}$ & 0 & 0 \\
\hline $3 x_{c}^{2}-r_{c}^{2}$ & 0 & 0 & $2 V\left(y_{a}, 3 x^{2}-r_{c}^{2}\right) g_{1}$ & $-V\left(y_{a}, 3 x^{2}-r_{c}^{2}\right) g_{2}$ & $-V\left(y_{a}, 3 x^{2}-r_{c}^{2}\right) g_{3}$ & 0 & 0 \\
\hline
\end{tabular}

$$
\begin{aligned}
& V\left(s_{a}, x y_{c}\right)=\frac{1}{\sqrt{3}} V_{s d \sigma} \\
& V\left(x_{a}, x y_{c}\right)=\frac{1}{3} V_{p d \sigma}+\frac{1}{3} \frac{1}{\sqrt{3}} V_{p d \pi}, \\
& V\left(x_{a}, y z_{c}\right)=\frac{1}{3} V_{p d \sigma}-\frac{2}{3} \frac{1}{\sqrt{3}} V_{p d \pi}
\end{aligned}
$$

TABLE III. 13-orbital tight-binding Hamiltonian matrix. 


$$
\begin{aligned}
& V\left(y_{a}, y^{2}-z_{c}^{2}\right)=\frac{1}{\sqrt{3}} V_{p d \pi}, \\
& V\left(y_{a}, 3 x^{2}-r_{c}^{2}\right)=-\frac{1}{3} V_{p d \pi} .
\end{aligned}
$$

\section{APPENDIX B}

As discussed in the text, our first priority in fitting the tight-binding Hamiltonian is to reproduce the available experimental data. A second priority is to fit the remaining important features of the bands obtained in the pseudopotential calculations of Refs. 12 and 14.

We first take

$$
V\left(x_{c}, y z_{c}\right)=0
$$

There are three reasons for doing this: (i) Preliminary fits indicated that this parameter should have a small value, of the order of $0.1 \mathrm{eV}$. This is to be expected because the coupling vanishes in the free $\mathrm{Cu}$ atom. (ii) Equation (B1) reduces the band fitting to an entirely linear procedure, with none of the ambiguities that can plague nonlinear fits. (iii) The impurity problem is reduced to the solution of scalar equations. With $V\left(x_{c}, y z_{c}\right) \neq 0$, the impurity problem on the cation site involves three $1 \times 1$ secular equations (corresponding to $s, d_{y^{2}-z^{2}}$, and $d_{3 x^{2}-r^{2}}$ electrons) and three $2 \times 2$ equations (corresponding to $p_{x}$ and $d_{y z}, p_{y}$ and $d_{x z}$, and $p_{z}$ and $d_{x y}$ electrons). But with $V\left(x_{c}, y z_{c}\right)=0$, the $p$ and $d$ electrons are decoupled, and this problem reduced to nine $1 \times 1$ problems.

The matrix elements $E(s, c), E(s, a), E(p, c)$, $E(p, a), E(d, c), E^{\prime}(d, c), V(s, s), V(x, x), V\left(x_{c}, y z_{c}\right)$, and $V\left(x_{a}, y z_{c}\right)$ are determined by fitting information about the band structure at $\Gamma$, the center of the Brillouin zone. According to group theory, there are three $\Gamma_{15}$ states. Their energies are determined by the equation:

$$
\left|\begin{array}{ccc}
E(p, a)-E & V(x, x) & -V\left(x_{a}, y z_{c}\right) \\
V(x, x) & E(p, c)-E & 0 \\
-V\left(x_{a}, y z_{c}\right) & 0 & E(d, c)-E
\end{array}\right|=0
$$

Experimentally ${ }^{16}$ the wave function at the top of the valence band $\left(\Gamma_{15}\right)$ is composed of $25 \% \mathrm{Cl} 3 p$, $75 \% \mathrm{Cu} 3 d$, and a negligible percentage of $\mathrm{Cu} 4 p$. To eliminate the $\mathrm{Cu} 4 p$ contribution at the valence-band maximum, we first choose $V(x, x)$ $=0$. The remaining off-diagonal parameter $V\left(x_{a}, y z_{c}\right)$ is then fixed by requiring that the $25 \%$ $\mathrm{Cl} 3 p$ and $75 \% \mathrm{Cu} 3 d$ character be reproduced. The diagonal matrix elements $E(p, a)$ and $E(d, c)$ are chosen so that the energy at the top of the valence band is zero and the lower $\mathrm{Cl} 3 p$-like valence band (also $\Gamma_{15}$ ) occurs at $-5 \mathrm{eV}$, in agreement with photoemission data. This leaves $E(p, c)$ to be determined.

The matrix element $E^{\prime}(d, c)$ can be determined by the position of peak $B$ of the photoemission data (Fig. 2), which corresponds to the $\Gamma_{12}$ valence band. The matrix elements $E(s, c), E(s, a)$, and $V(s, s)$ determine the bottoms of the conduction band and the lowest valence band, both of which are $\Gamma_{1}$. The bottom of the conduction band is given by the experimental band gap $(3.25 \mathrm{eV})$, and the bottom of the lowest valence band is taken from the calculation of Kleinman and Mednick.

\begin{tabular}{|c|c|c|c|c|c|}
\hline$z_{c}$ & $x_{c} y_{c}$ & $y_{c} z_{c}$ & $x_{c} z_{c}$ & $y_{c}^{2}-z_{c}^{2}$ & $3 x_{c}^{2}-r_{c}^{2}$ \\
\hline$-V\left(s_{a}, x_{c}\right) g_{3}^{*}$ & $V\left(s_{a}, x y_{c}\right) g_{3}^{*}$ & $V\left(s_{a}, x y_{c}\right) g_{1}^{*}$ & $V\left(s_{a}, x y_{c}\right) g_{2}^{*}$ & 0 & 0 \\
\hline 0 & 0 & 0 & 0 & 0 & 0 \\
\hline$V(x, y) g_{2}^{*}$ & $-V\left(x_{a}, x y_{c}\right) g_{2}^{*}$ & $-V\left(x_{a}, y z_{c}\right) g_{0}^{*}$ & $-V\left(x_{a}, x y_{c}\right) g_{3}^{*}$ & 0 & $2 V\left(y_{a}, 3 x^{2}-r_{c}^{2}\right) g_{1}^{*}$ \\
\hline$V(x, y) g_{1}^{*}$ & $-V\left(x_{a}, x y_{c}\right) g_{1}^{*}$ & $-V\left(x_{a}, x y_{c}\right) g_{3}^{*}$ & $-V\left(x_{a}, y z_{c}\right) g_{0}^{*}$ & $-V\left(y_{a}, y^{2}-z_{c}^{2}\right) g_{2}^{*}$ & $-V\left(y_{a}, 3 x^{2}-r_{c}^{2}\right) g_{2}^{*}$ \\
\hline$V(x, x) g_{0}^{*}$ & $-V\left(x_{a}, y z_{c}\right) g_{0}^{*}$ & $-V\left(x_{a}, x y_{c}\right) g_{2}^{*}$ & $-V\left(x_{a}, x y_{c}\right) g_{1}^{*}$ & $V\left(y_{a}, y^{2}-z_{c}^{2}\right) g_{3}^{*}$ & $-V\left(y_{a}, 3 x^{2}-r_{c}^{2}\right) g_{3}^{*}$ \\
\hline 0 & 0 & $V\left(x_{c}, y z_{c}\right)$ & 0 & 0 & 0 \\
\hline 0 & 0 & 0 & $V\left(x_{c}, y z_{c}\right)$ & 0 & 0 \\
\hline$E(p, c)$ & $V\left(x_{c}, y z_{c}\right)$ & 0 & 0 & 0 & 0 \\
\hline$V\left(x_{c}, y z_{c}\right)$ & $E(d, c)$ & 0 & 0 & 0 & 0 \\
\hline 0 & 0 & $E(d, c)$ & 0 & 0 & 0 \\
\hline 0 & 0 & 0 & $E(d, c)$ & 0 & 0 \\
\hline 0 & 0 & 0 & 0 & $E^{\prime}(d, c)$ & 0 \\
\hline 0 & 0 & 0 & 0 & 0 & $E^{\prime}(d, c)$ \\
\hline
\end{tabular}
The third of these parameters is fixed by requiring the lowest valence band to have $90 \% \mathrm{Cl} 3 \mathrm{~s}$ charac-

TABLE II. (Continued.) 
ter, as calculated by Zunger and Cohen. ${ }^{14}$

The matrix element $E(p, c)$ is fitted to the previously calculated results for the highest conduction bands $\left(\Gamma_{15}\right)$ at $\Gamma{ }^{12,14}$ The remaining matrix elements are determined by fitting previously calculated results at the $X$ point. In our model, the secular equations at the $X$ point are

$$
\left|\begin{array}{ccc}
E(s, c)-E & i V\left(x_{a}, s_{c}\right) & 0 \\
i V\left(x_{a}, s_{c}\right) & E(p, a)-E & i \frac{2}{3} V_{p d \pi} \\
0 & -i \frac{2}{3} V_{p d \pi} & E^{\prime}(d, c)-E
\end{array}\right|=0
$$

for $X_{1}$,

$$
E^{\prime}(d, c)-E=0,
$$

for $X_{2}$,

$$
\left|\begin{array}{ccc}
E(s, a)-E & i V\left(x_{c}, s_{a}\right) & i V_{s d \sigma} / \sqrt{3} \\
-i V\left(x_{c}, s_{a}\right) & E(p, c)-E & 0 \\
-i V_{s d \sigma} / \sqrt{3} & 0 & E(d, c)-E
\end{array}\right|=0,
$$

for $X_{3}$, and

$$
\left|\begin{array}{ccc}
E(p, c)-E & i V(x, y) & 0 \\
-i V(x, y) & E(p, a)-E & i\left(\frac{1}{3} V_{p d \sigma}+\frac{1}{3} \frac{1}{\sqrt{3}} V_{p d \pi}\right) \\
0 & -i\left(\frac{1}{3} V_{p d \sigma}+\frac{1}{3} \frac{1}{\sqrt{3}} V_{p d \pi}\right) & E(d, c)-E
\end{array}\right|=0
$$

for $X_{5}$. From these equations, the off-diagonal matrix elements can be determined directly:

$$
\begin{aligned}
& {\left[V\left(x_{a}, s_{c}\right)\right]^{2}=-\frac{\left[E(s, c)-X_{1}(1)\right]\left[E(s, c)-X_{1}(2)\right]\left[E(s, c)-X_{1}(3)\right]}{E(s, c)-E^{\prime}(d, c)},} \\
& \left(\frac{2}{3} V_{p d \pi}\right)^{2}=-\frac{\left[E^{\prime}(d, c)-X_{1}(1)\right]\left[E^{\prime}(d, c)-X_{1}(2)\right]\left[E^{\prime}(d, c)-X_{1}(3)\right]}{E(s, c)-E^{\prime}(d, c)}, \\
& {\left[V\left(x_{c}, s_{a}\right)\right]^{2}=-\frac{\left[E(p, c)-X_{3}(1)\right]\left[E(p, c)-X_{3}(2)\right]\left[E(p, c)-X_{3}(3)\right]}{E(p, c)-E(d, c)},} \\
& \left(V_{s d \sigma}^{2} / 3\right)=-\frac{\left[E(d, c)-X_{3}(1)\right]\left[E(d, c)-X_{3}(2)\right]\left[E(d, c)-X_{3}(3)\right]}{E(p, c)-E(d, c)}, \\
& {[V(x, y)]^{2}=-\frac{\left[E(p, c)-X_{5}(1)\right]\left[E(p, c)-X_{5}(2)\right]\left[E(p, c)-X_{5}(3)\right]}{E(p, c)-E(d, c)},}
\end{aligned}
$$

and

$$
\left(\frac{1}{3} V_{p d \sigma}+\frac{1}{3} \frac{1}{\sqrt{3}} V_{p d \pi}\right)^{2}=-\frac{\left[E(d, c)-X_{5}(1)\right]\left[E(d, c)-X_{5}(2)\right]\left[E(d, c)-X_{5}(3)\right]}{E(p, c)-E(d, c)} .
$$

Here $X_{i}(j)$, with $i=1,3$, and 5 and $j=1,2$, and 3, are the three energy values of the $X_{i}$ representation. We choose the $X_{i}(j)$ employing the following two conditions: (i) they should give a band structure which is in good agreement with previous cal- culations, and (ii) the tight-binding Hamiltonian should give a positive charge on the $\mathrm{Cu}$ site. Table I presents the parameters determined by this scheme. 
"Permanent address: Department of Physics, University of Science and Technology of China, Hefei, China. Please send reprint requests to the Physics Department Reprint Secretary, Urbana.

†Permanent address: Department of Physics, Texas A\&M University, College Station, Texas 77843.

${ }^{1}$ N. B. Brandt, S. V. Kuvshinnikov, A. P. Rusakov, and M. Semyonov, Zh. Eksp. Teor. Fiz. Pis'ma Red. 27, 37 (1978).

${ }^{2}$ C. W. Chu, A. P. Rusakov, S. Huang, S. Early, T. H. Geballe, and C. Y. Huang, Phys. Rev. B 18,2116 (1978).

${ }^{3}$ I. Lefkowitz, J. S. Manning, and P. E. Bloomfield, Phys. Rev. B 20, 4506 (1979).

${ }^{4}$ I. Lefkowitz (unpublished).

${ }^{5}$ C. I. Yu, T. Goto, and M. Veta, J. Phys. Soc. Jpn. 34, 693 (1973).

${ }^{6} \mathrm{M}$. Combescot and C. Benoit a la Guillaume, Phys. Rev. Lett. 44, 182 (1980).

${ }^{7}$ D. C. Reynolds, R. J. Almasey, C. W. Litton, G. L. Koos, A. B. Kunz, and T. C. Collins, Phys. Rev. Lett 44, 204 (1980).

${ }^{8}$ H. P. Hjalmarson, P. Vogl, D. J. Wolford, and J. D. Dow, Phys. Rev. Lett. 44, 810 (1980).

${ }^{9}$ O. F. Sankey, H. P. Hjalmarson, J. D. Dow, D. J. Wolford, and B. G. Streetman, Phys. Rev. Lett. 45, 1656 (1980).

${ }^{10}$ R. E. Allen and J. D. Dow, J. Vac. Sci. Technol. 19,
383 (1981).

${ }^{11}$ W. A. Harrison, Electronic Structure and the Properties of Solids (Freeman, San Francisco, 1980).

${ }^{12}$ L. Kleinman and K. Mednick, Phys. Rev. B 20, 2487 (1979).

${ }^{13}$ A. B. Kunz, R. S. Weidman, and T. C. Collins, Int. J. Quantum Chem. Symp. 13, 453 (1979).

${ }^{14}$ A. Zunger and M. L. Cohen, Phys. Rev. B 20, 1189 (1979).

${ }^{15}$ H. Müller, S. Ves, H. D. Hoehheimer, M. Cardona, and A. Jayaraman, Phys. Rev. B 22, 1052 (1980).

${ }^{16}$ A. Goldmann, Phys. Status Solidi B 81,9 (1977).

${ }^{17}$ G. van der Laan, G. A. Sawatzky, C. Haas, and H. W. Myron, Phys. Rev. B 20, 4287 (1979).

${ }^{18}$ For the highly covalent group-IV and III $-\mathrm{V}$ materials of Ref. 8, the choice of constants was $\beta_{s}=0.8$ and $\beta_{p}=0.6$. [See P. Vogl, H. P. Hjalmarson, and J. D. Dow (unpublished)]. For the more ionic I-VII materials of interest in the present paper, we choose $\beta_{s}=0.95, \beta_{p}=0.90$, and $\beta_{d}=1.00$.

${ }^{19}$ S.-Y. Ren (unpublished).

${ }^{20}$ A. Goltzene, O. Scherab, B. Meyer, and S. Nikitine, Opt. Commun. 5, 248 (1972).

${ }^{21}$ A. Goltzene, B. Meyer, C. Schwab, and K. Cho, Phys. Status Solidi B $\underline{69}, 237$ (1975).

${ }^{22}$ A. Goltzene and C. Schwab, Phys. Status Solidi B 71, K67 (1975). 